\title{
Digital Image Correlation technique applied to mechanical characterisation of aluminium foam
}

\author{
M. De Giorgi ${ }^{\mathrm{a}}$, S. Giancane, R. Nobile, and F. Palano \\ Dipartimento di Ingegneria dell'Innovazione, Università del Salento, Via per Monteroni, 73100, \\ Lecce, Italy
}

\begin{abstract}
In this paper the possibility to employ the Digital Image Correlation (DIC) technique for the mechanical behaviour analysis of metallic foam was investigated. Image Correlation allowed to measure displacement and strain fields on closed and open cells aluminium foam specimens under two different loading conditions (compression and shear) and to characterise, with opportune calculation, information on the mechanical behaviour of foams. The adopted technique is suitable to conduct a deep analysis and also to appreciate the local heterogeneities that appear in a specimen during test. The parameters obtained with DIC analysis are confirmed by the global data obtained by testing machine proving the adopted methodology represents a valid tool for the study of these new materials.
\end{abstract}

\section{Introduction}

Aluminium foam constitutes a new class of material having a porous structure that confers them interesting mechanical properties and high potentiality in advanced engineering applications. Cellular structures consist of a solid and a gaseous phases and their cells morphology identifies two types of foams: open and closed cells foam.

Metal foams are useful in a variety of applications, due to their unique properties: low density, high strength to weight ratio, high damping capacity, exceptional energy absorption ability, high thermal conductivity, good electrical conductivity and good corrosion resistance. Due to high strength to weight ratios, these materials are appropriate for lightweight structural applications. Nevertheless, the applications are yet limited since the mechanical characterisation of these new materials is still insufficient and incomplete. However, before potential applications can be realized, a thorough understanding of the mechanical behaviour of these materials is required.

Anyway, a rich literature about the mechanical characterisation under uniaxial load is available [1-10]; on the contrary the works concerning the shear behaviour of foam are quite poor. As evident from scientific literature [11-15], knowledge of the foam shear behaviour is yet incomplete because it is limited to some typologies of foams and because a consolidate methodology for shear characterisation does not yet exist.

The aluminium foams can present highly localised strain phenomena that evolve in space and in time. Therefore, macroscopic mechanical properties, as usually determined by standard tests, are

\footnotetext{
a e-mail: marta.degiorgi@unisalento.it
} 
generally strongly influenced by statistical cell distribution, making a difficult task to develop reliable analytical framework. For this reason, it results relevant the need to develop a database as large as possible of the mechanical characteristics of various foam typologies having different morphology, density and thickness. Moreover, the study of the foam in-homogeneity effect on mechanical properties would be highly facilitated by the use of a full-field experimental technique for the monitoring of displacement or strain.

In this work, Image Correlation has been employed to measure displacement and strain field on aluminium foam specimens in order to characterise mechanical behaviour under compressive and shear loading conditions.

Digital Image Correlation (DIC) is a full-field and non-contact technique for the measurement of displacements and strain. It is based on pattern recognition and exploits the correspondence between the markers of a virtual grid superimposed on the first images. Considering a batch of images, the first is taken as reference and a virtual grid is superimposed on it; opportune algorithms allow to find the position of the markers of the grid for every image and to calculate the field of superficial displacements at the time which an image is referred to. The images must present a significant contrast to make the process working.

DIC is an appealing technique for studying the heterogeneities that evolve into a material during a quasi-static or dynamic load application, because under opportune conditions it shows a precision and versatility that is difficult to get employing other techniques $[16,17]$. When compared with the special requirement of traditional optical measurements, DIC is a versatile method because it takes advantage of the natural speckle pattern on the specimen surface and needs a common camera to store the images to be elaborated later.

Some data are available in scientific literature about the application of Digital Image Correlation method investigate cellular materials.

Zhoua et al. [18] present a study on the effects of heat treatment on compressive behaviour of open cell aluminium foams in unperturbed, annealed and strengthened conditions, using a combination of mechanical testing and Digital Image Correlation technique. The significant effects of heat treatments on foam compressive strengths, stress-strain behaviour and strain mechanisms are attributed to the changes of the microstructure and micro-scale mechanical properties.

Wang et al. [19] employed image correlation to reveal the evolution of the strain texture on the surface of polyurethane open cell foam samples with different densities. The results support the interpretation that the collapse of light open cell foams occurs as a phase transition phenomenon.

Jin et al. [20] studied the heterogeneous deformation of a closed cell polyurethane foam and strain concentration at the foam-steel interface were obtained using the 3-dimensional Digital Image Correlation (3D-DIC) technique.

Bastawros et al. [21] investigated the evolution of plastic strain in a cellular aluminium alloy upon axial compression through a Digital Image Correlation procedure. Three stages in the deformation response were identified: localized plastic strain at cell nodes; discrete bands of concentrated strain containing cell membranes, complete plastic collapse of one band.

An increased understanding of the strain localization in Alporas aluminium foam subject to uniaxial compression is achieved in [22] by Werther et al. via a dual camera data acquisition system, used to observe adjacent specimen faces. DIC is employed to investigate local strain associated with the compaction process. Theoretical predictions are developed using a bifurcation approach to localization, which successfully predicted compaction in aluminium foam.

Issen et al. [23] employed surface strain mapping to examine the onset of localized compaction and experimental observations are compared with theoretical predictions for band formation, developed using bifurcation theory. Uniaxial compression tests on aluminium foam reveal formation of bands of localized compaction, approximately perpendicular to the axial compression direction, consisting of predominantly axial strain and resulting from cell collapse.

Even if DIC was largely applied for compressive characterization of foams, no significant employment of this full field technique to study the shear behaviour of foam appears in literature. The aim of this work is to give a more exhaustive sight to the possibility that DIC offers in studying 
metallic foam. A significant number of tests were performed and the DIC data were compared with the results taken from test machine data, showing a remarkable agreement.

Analysed aluminium foams were Alporas closed cells and M-Pore open cells foams. All the tests were performed using a servo-hydraulic testing machine: the compressive tests were carried out positioning the specimen between two plates gripped on the machine, while shear loading conditions were obtained using a purposely designed device for biaxial load transmission.

Images in an 8-bit format (256 grey levels) were stored during compressive and shear tests employing a $\mathrm{CCD}$ and then were opportunely elaborated to determine the displacements and the strains field.

\section{Materials and methods}

Experimental characterisation is carried out through shear tests and uniaxial compression tests in two different directions: foaming direction and perpendicular direction to foaming.

Each specimen was recorded during loading test by a RDT/1 CCD camera (by DRS Technology) using a rate of 1.25 image per second and a spatial resolution of about $0.1 \mathrm{~mm} /$ pixel. For an efficient image correlation the images must present a gray level pattern usually obtained by means of paintings. In the case of aluminium foams, the surface intrinsically appears with a strong pattern of "grains" due to the presence of cells or due to the rough morphology of external skins. This allows avoiding any further operation for preparing the specimens and at the same time it guarantees an excellent correlation even for large displacements.

Open and closed aluminium foams were analysed. Open cells foam produced by M-Pore $\mathrm{GmbH}$ in Dresden (Germany), here named "M-PORE", presents porosity of 20 ppi (pores per inches) and is produced in plates having thickness of $30 \mathrm{~mm}$. Closed cells foam produced by Gleich $\mathrm{GmbH}$ in Germany, here named "ALPORAS", presents porosity about $250 \mathrm{~kg} / \mathrm{m}^{3}$ and is produced in plates $30 \mathrm{~mm}$ thick.

All tests were performed using a servo-hydraulic machine MTS having load capacity of $100 \mathrm{kN}$.

\subsection{Compression tests}

Cutting of foam sheets has been carried out through a circular sawing machine. The density of each specimen was calculated measuring its mass by a balance having precision of 0.01 grams and its dimensions by a centesimal calliper.

For each test, the values of force and displacement of the actuator were recorded. The applied stress is obtained dividing the force for the cross-section of each specimen, while axial strain is calculated dividing the actuator displacement for the specimen initial thickness. Compression tests were interrupted when a reduction of about $70 \%$ of initial thickness was reached in order to highlight the densification phases of foam.

All foams, whatever is the production method, show a typical behaviour under compression: for small strain there is an increment of stress that appears as elastic, but, after an accurate analysis, it emerges that the material deformation process is only partly reversible, because the stress concentration due to imperfection of foam originates localised and diffuse plastic strain. Because of this behaviour, otherwise from homogeneous materials, the elastic modulus of metal foams cannot be calculated from the slope of the curve in the first phases of compression test. The accepted procedure suggests loading the foam specimen up to $75 \%$ of $\sigma_{\text {plateau }}$, defined in a preliminary test, and then to gradually remove the load; when the specimen results completely unloaded, the load is increased again up to complete densification; E value is assumed as the slope of unloading curve. After the initial increment of stress up to a plateau stress, $\sigma_{\text {plateau }}$, a macroscopic foam plastic collapse appears, recognizable by a relevant slope decrease of stress-strain curve. After an extended plateau the curve gradually evolved in densification regime; now the walls of cells come in contact and the stiffness abruptly increases [1-10]. 


\section{EPJ Web of Conferences}

Compressive tests, to determine the $\sigma_{\text {plateau }}$, were performed in previous works [10]. In this study, an automatic procedure to load-unload-reload specimens was implemented according to following criteria:

- load phases until $75 \% \sigma_{\text {plateau }}$ and unload using the force control mode, with test rate variable from $4.3 \mathrm{~N} / \mathrm{s}$ to $9.4 \mathrm{~N} / \mathrm{s}$ depending on foam typology and load direction;

- reload phase using displacement control mode whit test rate equal to $0.03 \mathrm{~mm} / \mathrm{s}$.

The cross section specimen dimensions were:

- tests in direction of foaming: $30 \times 15 \mathrm{~mm}$;

- tests in normal direction of foaming 40x15 mm.

Testing machine was equipped with two stiff plates and foam specimen is positioned between them at the test beginning (Figure 1).

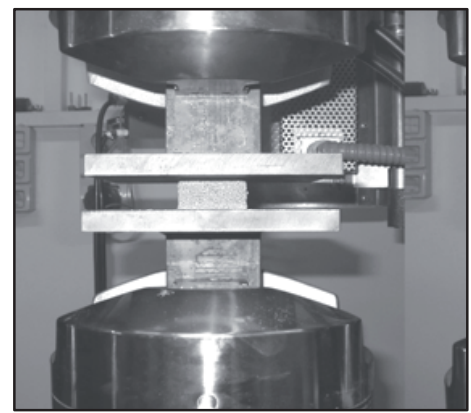

Fig. 1. Compression test.

\subsection{Shear tests}

Shear tests were conducted using a purposely-designed device (Figure 2a) and a cruciform specimen (Figure $2 b$ ) to guarantee shear conditions during load transmission according to Mohr's circle theory (Figure 2c) as discussed in [24]. It applied simultaneously a tensile stress by the vertical arms gripped in the MTS machine and a compressive stress by means of the horizontal arms on a cruciform specimen.

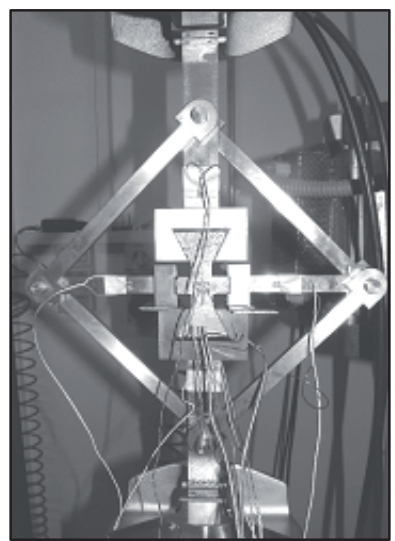

(a)

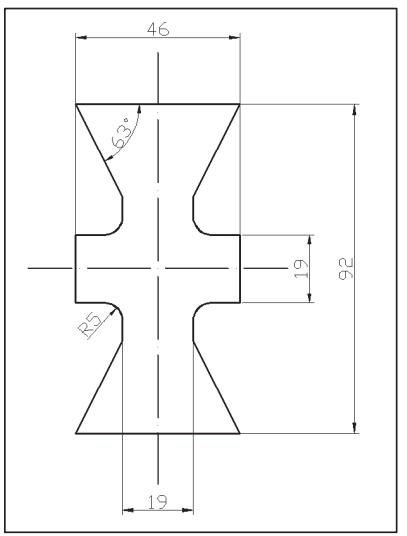

(b)

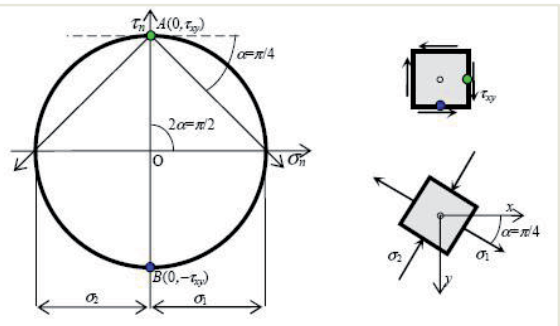

(c)

Fig. 2. Shear test (a) and shear specimen (b) 


\section{Results and discussion}

A significant number of tests were carried on and both typologies of foams were considered. In particular, 4 shear tests and 8 compressive tests were conducted. Every specimen presents a unique ID "X-YY-(Z)-n", composed by a first letter "X" that indicated the type of test (C for compression, $S$ for shear), two letters "YY" that indicated the type of foam (AL for Alporas and MP for M-pore), for compression test only a letter " $\mathrm{Z}$ " indicated the load direction ( $\mathrm{F}$ for foaming and $\mathrm{N}$ for normal), and an increasing numerical index "n". For all tests, firstly the MTS data of force has been triggered and synchronised with the acquired images; moreover, averaging the strain data of each map, a comparison between DIC data and MTS data is presented.

\subsection{Compression tests}

As example, the results of compression test on specimen C-AL-N-4 are showed in Figure 3. What firstly emerges is the excellent correspondence between displacement and strain data of MTS and the data extracted from DIC analysis.
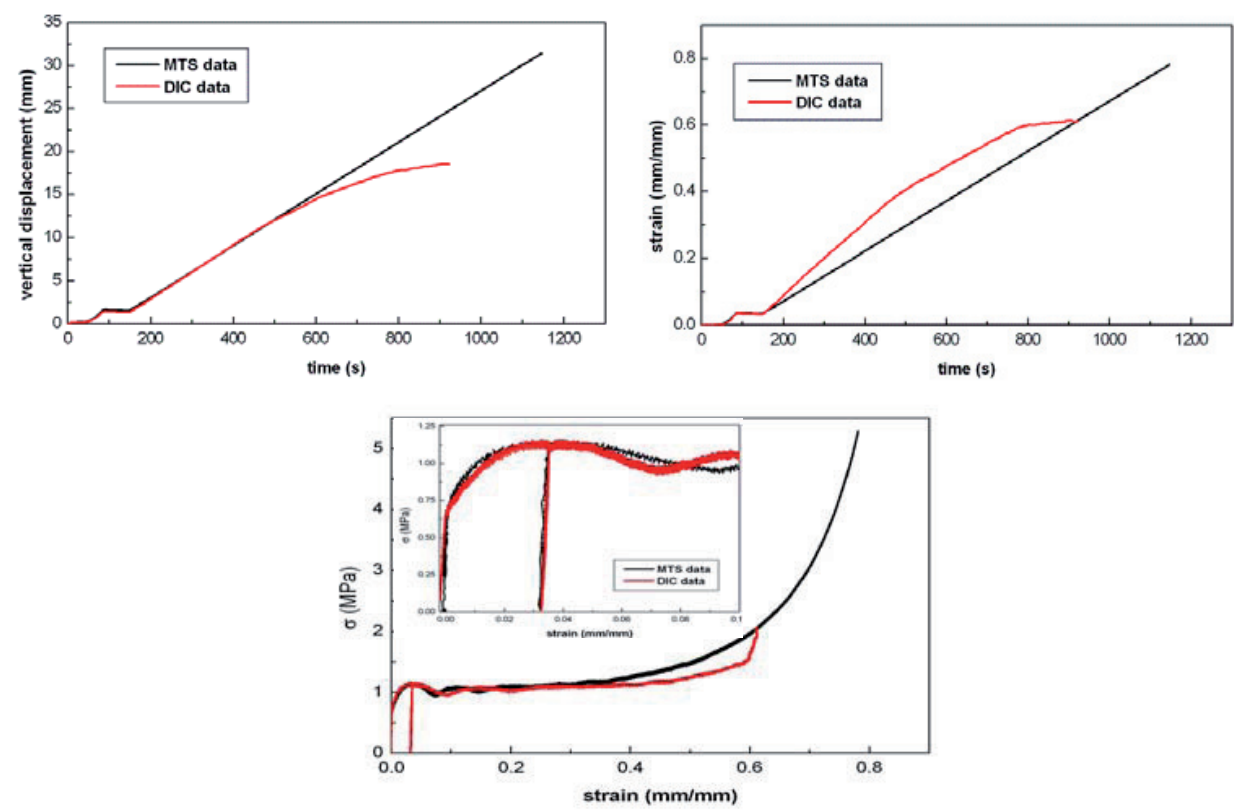

(a)

(c)

(b)

Fig. 3. Comparison between results for test C-AL-N-4, obtained from MTS data (in red line) and from DIC elaboration (in black line) respectively for displacements (a), strain (b) and $\sigma-\varepsilon$ curves (c).

From the elaboration, it can be observed that at time $\mathrm{t}=500 \mathrm{~s}$ correlation become less precise and "DIC displacement" differs more and more from the "MTS displacement". It can be explained because of the relevant strain that the specimen undergoes during test: in the final part of the test, in fact, the original aspect of surface is completely lost causing a growing difficult for DIC algorithm to recognize the marker movements. This occurrence does not represent an obstacle in using DIC for compression characterisation, because the most important part of the curve is completely and faithfully reconstructed starting from digital 2D image; in Figure 3c the accuracy of DIC elaboration can be appreciated. The advantage of a DIC analysis resides in the possibility to have information about the local heterogeneities that evolve during test; in fact, even for test C-AL-N-4, the strain 
maps (see fig. 4) show a non-uniform development of compressive strain and in particular a zone on the lower right corner appears to be the most significant since the beginning of test. This confirms that the behaviour of the aluminium foam is strongly heterogeneous. In Table 1, results for compressive test are exposed.
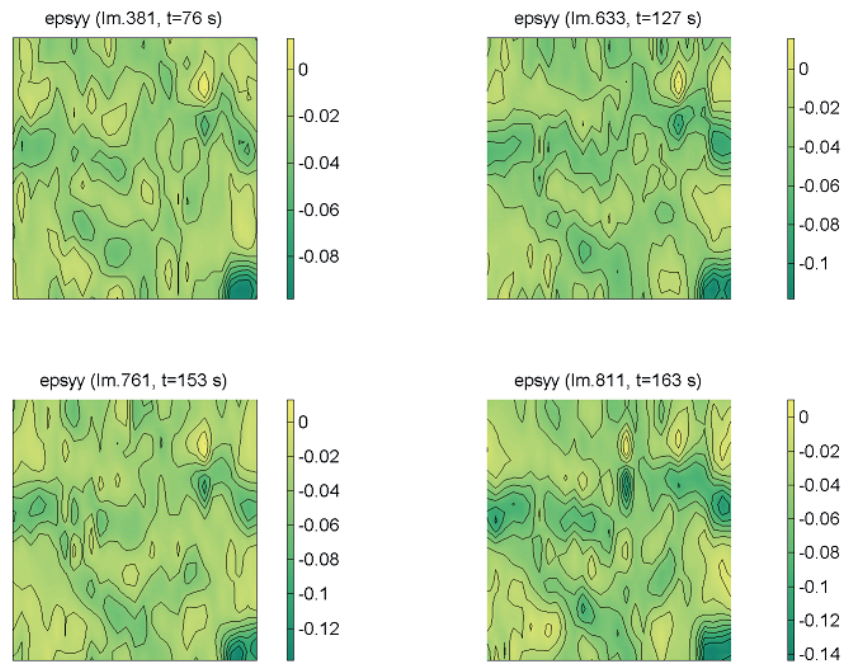

Fig. 4. Strain maps at different time of the test C-AL-N-4.

Tab. 1. Results for compressive test

\begin{tabular}{|c|c|c|c|c|}
\hline $\begin{array}{c}\text { Foam } \\
\text { typology }\end{array}$ & ID & Density $\left[\mathrm{kg} / \mathrm{m}^{3}\right]$ & $\mathbf{E}_{\text {unload }}\left[\mathrm{N} / \mathrm{mm}^{2}\right]$ & $\sigma_{\text {plateau }}\left[\mathrm{N} / \mathrm{mm}^{2}\right]$ \\
\hline \multirow{4}{*}{ 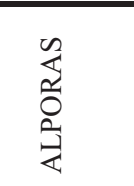 } & C-AL-F-1 & 250 & 745 & 1.76 \\
\hline & C-AL-F-2 & 249 & 670 & 1.64 \\
\hline & C-AL-N-3 & 236 & 402 & 1.23 \\
\hline & C-AL-N-4 & 217 & 396 & 1.14 \\
\hline \multirow{4}{*}{$\begin{array}{l}\text { 1) } \\
\frac{1}{0} \\
0 \\
\overbrace{1}^{1}\end{array}$} & C-MP-F-1 & 210 & 350 & 1.02 \\
\hline & C-MP-F-2 & 217 & 355 & 1.11 \\
\hline & C-MP-N-3 & 213 & 153 & 0.95 \\
\hline & C-MP-N-4 & 253 & 261 & 1.13 \\
\hline
\end{tabular}

\subsection{Shear tests}

For test S-MP-1, the images were elaborated in the straight position (original) and also as rotated with an angle of $45^{\circ}$ in order to verify the hypothesis on the basis of the device for load application. Therefore shear strain was also calculated according to Mohr theory as $\gamma=\varepsilon_{y y}-\varepsilon_{x x}$, the $\tau-\gamma$ curves are obtained for the two batches of images (original and rotated) and then compared with the ones extracted from MTS data (see Fig. 5).

It is possible to observe that the rupture was effectively provoked by shear crack (Fig. 5b). In fact DIC analysis allowed highlighting the presence of a strong shear band crossing through the specimen and absorbing the main displacement imposed by test machine (Fig 5a and 5c). Calculations of shear strain $\gamma$ carried out on original images and on the $45^{\circ}$ tilted ones present a remarkable coincidence. In the same graph, the comparison between the $\tau-\gamma$ curves obtained from MTS data and DIC results are 
reported (see Fig. 5d). The figure clearly highlights the agreement between DIC data and MTS data and the different windows chosen for monitoring the evolution of test cause the light divergence of the results. In Table 2, results for shear test are exposed.

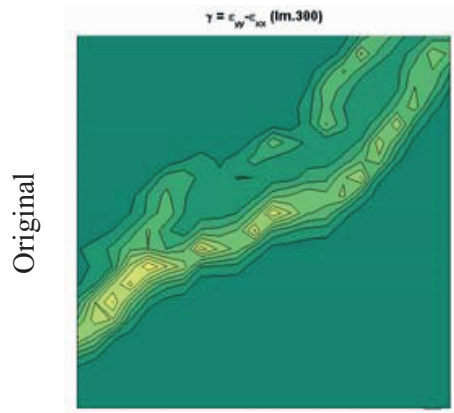

a)

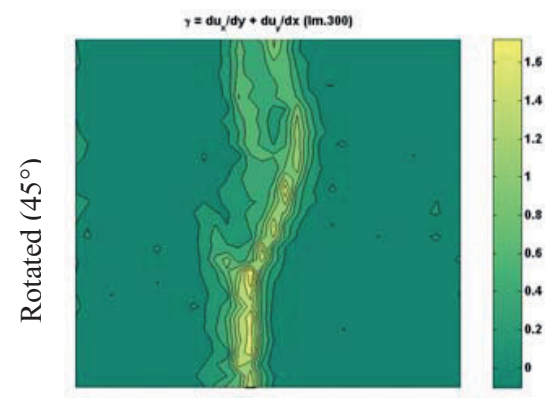

c)

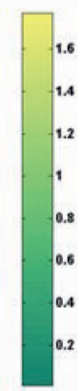

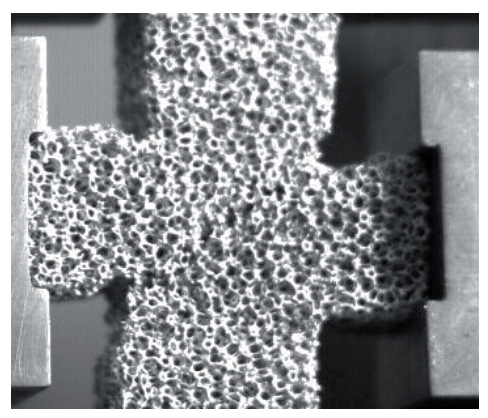

b)

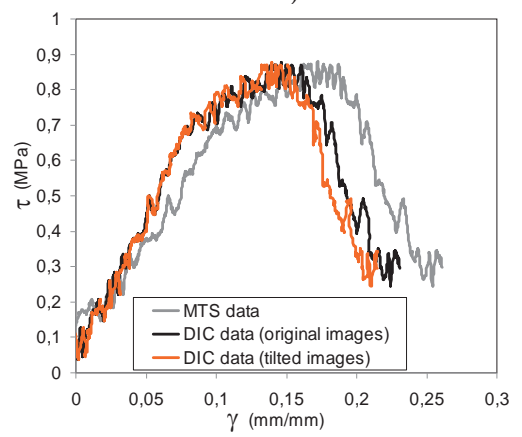

d)

Fig. 5. Strain maps and $\tau-\gamma$ curves obtained on M-pore specimen.

Tab. 2. Results for shear test

\begin{tabular}{ccccc}
\hline Foam & ID & Density $\left[\mathrm{kg} / \mathrm{m}^{3}\right]$ & $\mathbf{G}\left[\mathrm{N} / \mathrm{mm}^{2}\right]$ & $\tau_{\max }\left[\mathrm{N} / \mathrm{mm}^{2}\right]$ \\
typology & & & & \\
\hline \multirow{2}{*}{ ALPORAS } & S-AL-1 & 207 & 6.46 & 0.83 \\
\cline { 2 - 5 } & S-AL-2 & 235 & 4.90 & 1.26 \\
\hline \multirow{2}{*}{ M-PORE } & S-MP-1 & 251 & 5.33 & 0.88 \\
\cline { 2 - 5 } & S-MP-2 & 227 & 5.22 & 0.85 \\
\hline
\end{tabular}

\section{Conclusions}

In this work, DIC technique was applied as a full-field method to measure the strain field originated by compressive and shear load in aluminium foam specimens. The comparison between testing machine and DIC data showed a great accordance. Therefore, the DIC is revealed to be an efficient and universal methodology to study the heterogeneities that develop in a cellular material during the quasi-static load application. This technique, under particular conditions, shows a precision and a versatility that is difficult to obtain with other experimental method. 


\section{References}

1. E. Andrews, W. Sanders, L.J Gibson, Compressive and tensile behavior of aluminum foams, Mater Sci and Eng A270, 113-124 (1999).

2. M.F. Ashby, A.G. Evans, N.A. Fleck, L.J. Gibson, J.W. Hutchinson, H.N.G. Wadley, Metal Foams: A Design Guide, Butterworth-Heinemann publications (2000).

3. J. Banhart, J. Baumeister, Deformation characteristics of metal foam, J of Mater Sci, 33, 14311440 (1998).

4. Y. Sugimura, J. Meyer, M.Y. He, H. Bart-Smith, J. Grenstedt, A.G. Evans, On the mechanical performance of closed cell Al alloy foams, Acta Mater., 45, 5245-5259 (1997).

5. T.J. Lu, J.M. Ong, Characterization of close-celled cellular aluminum alloys, $J$ of Mater Sci, 36, 2773-2786 (2001).

6. L.J. Gibson, M.F. Ashby, Cellular Solids: Structure and Properties, 2nd edn, Cambridge University Press, UK (1997).

7. T.G. Nieh, K. Higashi, J. Wadsworth, Effect of cell morphology on the compressive properties of open-cell aluminium foam, Mater Sci and Eng, A283, 105-110 (2000).

8. K.Y.G. McCullough, N.A. Fleck, M.F. Ashby, Uniaxial stress-strain behaviour of aluminium alloy foams, Acta Mater., 47, No. 8, 2323-2330 (1999).

9. C. San Marchi, A. Mortensen, Deformation of open-cell aluminum foam, Acta Mater., 49, 3959-3969 (2001).

10. M. De Giorgi, A. Forte, R. Nobile, F. Palano, Caratterizzazione a compressione di schiume di Alluminio, XXXVII National Congress AIAS, 10-13 September 2008, Roma.

11. E.W. Andrews, G. Gioux, P. Onck, L.J. Gibson, Size effects in ductile cellular solids. Part II: experimental results, Int J of Mech Sci, 43, 701-713 (2001).

12. J.F. Rakow, A.M. Waas, Size effects and the shear response of aluminium foam, Mech Mat, 37, 69-82 (2005).

13. T.J. Lu, J.M. Ong, Characterization of close-celled cellular aluminum alloys, J. of Mater Sci, $36,2773-2786(2001)$.

14. J.S. Blazy, A. Marie-Louise, S. Forest, Y. Chastel, A. Pineau, A. Awade, C. Grolleron, Deformation and fracture of aluminum foams under proportional and non proportional multiaxial loading: statistical analysis and size effect, Int. J. Mech. Sci.,46, 217-244 (2004).

15. L. Peroni, M. Avalle, M. Peroni, The mechanical behavior of aluminum foam structures in different loading conditions, Int. J. of Impact Eng, 35, 644-658 (2008).

16. J. Abanto-Bueno, J. Lambros, Investigation of crack growth in functionally graded materials using digital image correlation, Eng Fract Mech, 69, 1695-1711(2002).

17. E.B. Li, A.K. Tieu, W.Y.D. Yuen, Application of digital image correlation technique to dynamic measurement of the velocity field in the deformation zone in cold rolling, Opt and Lasers in Eng, 39, 479-488 (2003).

18. J. Zhoua, Z. Gao, A.M. Cuitiño, W.O. Soboyejo, Effects of heat treatment on the compressive deformation behavior of open cell aluminum foams, Mater Sci and Eng A386, 118-128(2004).

19. Y. Wang, A.M. Coutiño, Full-field measurements of heterogeneous deformation patterns on polymeric foams using digital image correlation, Int J Solids and Struct, 39, 3777-3796 (2002).

20. H. Jin, W.-Y. Lu, S. Scheffel, T. D. Hinnerichs, M.K. Neilsen, Full-field characterization of mechanical behavior of polyurethane foams, Int J Solids and Struct, 44, 6930-6944 (2007).

21. A.F. Bastawros, H. Bart-Smith, A.G. Evans, Experimental analysis of deformation mechanisms in a closed-cell aluminum alloy foam, $J$ of the Mech and Phys of Solids, 48, 301-322 (2000).

22. D.J. Werther, A.J. Howard, J.P. Ingraham, K.A. Issen, Characterization and modeling of strain localization in aluminum foam using multiple face analysis, Scripta Mater, 54, 783-787 (2006).

23. K.A. Issen, T.P. Casey, D.M. Dixon, M.C. Richards, J.P. Ingraham, Characterization and modeling of localized compaction in aluminum foam, Scripta Mater, 52, 911-915 (2005).

24. V. Dattoma, M. De Giorgi, S. Giancane, R. Nobile, Studio del comportamento a taglio di schiume d'alluminio tramite analisi DIC, AIAS 2009 XXXVIII Convegno Nazionale, Torino, 9-11 Settembre 2009. 